\title{
Attractive Sulfur $\cdots \pi$ Interaction between Fluorinated Dimethyl Sulfur (FDMS) and Benzene
}

\author{
Shihai Yan, Sang Joo Lee, ${ }^{\dagger}$ Sunwoo Kang, Kwang-Hyun Choi, ${ }^{,}$Soon Ki Rhee, ${ }^{\circ+}$ and Jin Yong Lee \\ Department of Chemistry, Sunghyunkw University, Suwon $4+0-746$, Korea. ${ }^{*}$ E-mail jimpe askkuedu \\ Center for Computational Biologv and Bioinformatics, Korea Institute of Science and Technologv Information, \\ Iuseong, Daejeon 305-806, Korea \\ ${ }^{\ddagger}$ Department of Chemistry, Chonnam Vational Lniversity, Gwangin 500-757, Korea \\ Received November 14, 2006
}

\begin{abstract}
The benzene complexes with dimethyl sulfur (DMS) and fluorinated DMS (FDMS) have been investigated using ab initio calculations. The natural bond orbital $(\mathrm{NBO}$ ) charge population on $\mathrm{S}$ atom varies remarkably for different conformations of DMS and FDMS. which determines the possible binding modes for their benzene complexes. The electronegative substituent at the methyl group of DMS causes a significant change in the molecular electrostatic potential around the sulfur atom and changes the interaction mode with aromatic ring. It was found that the sulfur $\cdots \pi$ interaction mode does not occur in the DMS-benzene complex. while it does in the FDMS-benzene complex. Both B3LYP and MP2 methods provide reliable stnctures. while the interaction energy obtained by B3̈LYP is unreliable.
\end{abstract}

Key Words : Dimethyl sulfur (DMS), Fluorinated DMS. Sulfur- $\pi$ interaction, Molecular electrostatic potential

\section{Introduction}

Noncovalent interactions involving the aromatic side chains of certain amino acids are important factors in determining the dynamics of protein folding. The process of protein folding is governed mainly/partly by noncovalent interactions such as hydrogen-bonding hydrophobic and electrostatic interactions. which involve the aromatic side chains of phenylalanine tyrosine and tryptophan residues. ${ }^{1}$ Understanding the protein-protein interaction is emerging as critical aspects for the design of new drug and biosensors.' There are groups of alternating aromatic and sulfur-containing side chains that could stabilize proteins through strong noncovalent interactions between sulfur groups and the electron system of aromatic rings. and theoretical analysis illustrates that noncovalent interactions involving aromatic and sulfur containing side chains play a significant role in the early stages of protein folding by promoting the formation of $\alpha$-helices. ${ }^{3}$

Morgan and co-workers ${ }^{\dagger}$ first proposed that a strong. favorable interaction exists between aromatic rings and divalent sulfur atoms to elucidate the high frequency of contacts observed in protein crystal structures between sulfur-bearing amino acids (cysteine and methionine) and those include an ring (histidine tryptophan. tyrosine. and phenylalanine). Since then. the relevant experimental inspections ${ }^{5-15}$ theoretical studies ${ }^{1622}$ involving molecular mechanics and quantum chemical calculations as well as the database searches ${ }^{3-2-26}$ have been carried out. It is confirmed that the sulfur is more frequently found above the aromatic

${ }^{\mathrm{a}} \mathrm{KHC}$ is from the Department of Chemical Engineering. Sungkyunkwan University, and joined this research as an undergraduate research involvement progrann. rings than expected from the random association of amino acids. and such interaction is called "sulfur $\cdots \pi$ " $(\mathrm{S} \cdots \pi)$ interaction. Subsequently. the possible importance of the $S$ $\cdots \pi$ interaction is pointed out. A sulfur (S) atom can interact favorably with an aromatic ring via an $\mathrm{S} \cdots \pi$ interaction. which has been detected in the hydrophobic core and may contribute to protein stability. However. the origin of $S \cdots \pi$ interaction is not clarified yet. The most stable configurations place the $\mathrm{S}$ at a large elevation above the ring plane (as is in agreement with the protein data). but with the $\mathrm{S}$ lone pairs directed away from the ring centroid. an orientation impossible for interaction amino acids. ${ }^{13.25}$ Whereas it also has been pointed out that the interacting $S$ atom predominantly expresses an affinity towards the edge of the aromatic ring rather than interacts in a planar stacking fashion. Therefore. the usage of terminology of $S \cdots \pi$ interaction does not seem accurate.$^{23}$ Whether the genuine $S \cdots \pi$ interaction exists or not? This is indeed the case that the small binding energy $(\sim 1-2 \mathrm{kcal} / \mathrm{mol})^{25}$ of gas-phase sulfur-aromatic complex makes it a challenge for both experiment and theory. Although it has attracted much attention, the controversy is still going on.

It is well known that dimethyl sulfur (DMS). a product of biodegradation of organosulfur compounds in marine environments. plays a significant role in the atmospheric sulfur cycle. ${ }^{27-33}$ DMS is taken as one of the responsible sources for the acidity of atmosphere and formation of cloud. This molecule has been chosen as the model to estimate whether the $S \cdots \pi$ interaction is significant enough to play a role in determining protein structure ${ }^{3(1)}$ while the DMS orientation of the selected structures are not consistent with those reported in experiment or found in the Brookhaven Protein Data Bank ${ }^{34}$ and the Cambridge Crystallographic Database ${ }^{26}$ Exploration on $S \cdots \pi$ interaction of 
DMS-benzene system might provide significant contributions to the protein structure and the enzyme mechanisms in biological systems. First of all. one should be capable of understanding the structure, stability, and some other properties of the intermolecular clusters taking part in the reactions before the recognition about the reaction details occurring in atmospheric conditions. ${ }^{35}$ Thus. a high level quantum chemical calculation is needed to illuminate the origins of the frequently observed aromatic and sulfurcontaining amino acids interaction. Recently. a high level quantum chemical calculation on the complex of benzene and $\mathrm{H}_{2} \mathrm{~S}$ has been reported by Sherrill and coworkers. ${ }^{2}$ In their study. the interaction mode is more like the $\mathrm{H}^{\cdots} \pi$ interaction ${ }^{36.37}$ which has typically been seen in benzenewater dinmer. ${ }^{\text {jS.al }}$ Considering that most sulfur-containing compounds have the dimethyl units and the sulfur-bearing compounds in nature should include distinct steric volume in order to determine the effect on the preferred geometry and energy of the interaction, DMS should be considered as the simplest molecule for the study of $S \cdots \pi$ interaction. Therefore. DMS is chosen as a model molecule to represent the sulfur-containing amino acid side chain of the $\alpha$-helices and benzene served as the prototypical aromatic molecule as usual.

The primary motivation of this study is to obtain a reliable qualitative answer on the following two questions: (l) Whether the $S \cdots \pi$ interaction exists for genuine configurations found in proteins? (2) Is this interaction significant enough to play a role in determining the protein structure? The effect of molecular orientation of benzene ring and the geometry structure of DMS on $\mathrm{S} \cdots \pi$ interaction also has been inspected in this paper.

\section{Computational Methods}

The benzene carries $\pi$-electron density and the sulfur bears entpty d-orbitals, both of which are highly polarizable. Therefore. the dispersion interaction is expected to be significant. The $a b$ initio second-order Moller-Plesset perturbation theory (MP2) method includes an explicit treatment of electron correlation and perfonms better in deal with the dispersion interaction. The density functional theory (DFT) is a fundamentally different approach to the problem of electron correlation and has been successfully applied to a variety of problems. Especially the B3LYP hybrid density functional method is generally comparable to MP2 in performance with a distinct reduction in computational expense. Therefore. the optimizations of the DMS and benzene monomers as well as the inspection on the electronegative substituent effect in methyl groups of DMS are performed with MP2 and B3LYP methods with $6-311+\mathrm{G}^{*}$ basis set employing the Gaussian 03 program. ${ }^{41} \mathrm{MP} 2 / 6-311+\mathrm{G}^{*}$ calculations are reported to give reliable interaction energies for $S \cdots \pi$ interactions as compared with the state-of-the-art ab initio calculations. ${ }^{22}$ The $S \cdots \pi$ interaction energy of the DMS-benzene complex is detennined according to the following equation.
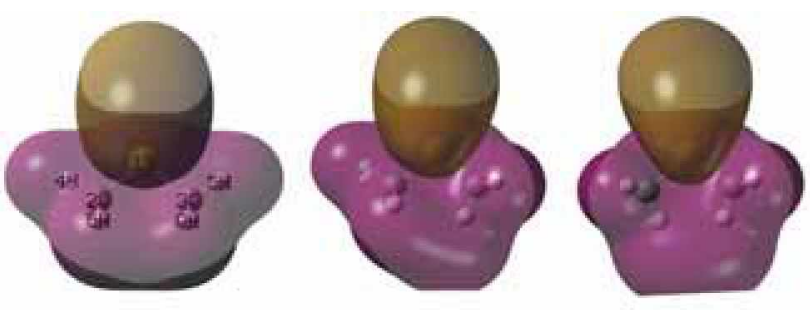

$\mathrm{C}_{2 \mathrm{v}}$
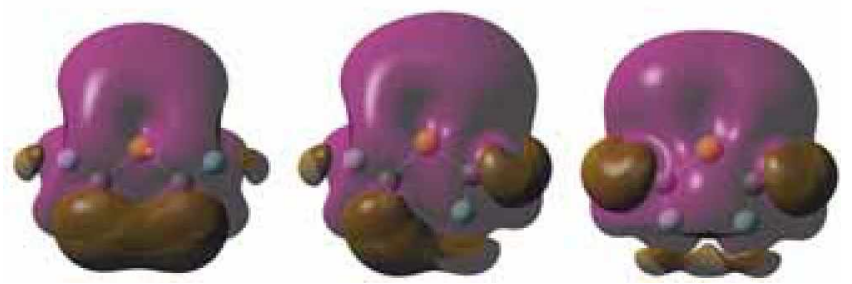

Figure 1. The electrostatic potential contour maps of DMS (upper row) and FDMS (lower row). The brown and purple color contours denote the negative and positive values, respectively.

$$
\Delta E=E_{\text {Complex }}-E_{\text {Benzzent }}-E_{\mathrm{DNS}}
$$

Here, $\Delta E$ denotes the $S \cdots \pi$ interaction energy between DMS and benzene. A negative $\Delta E$ implies an attractive interaction between DMS and benzene. Based on the optimized DMS and benzene monomer. six representative geometries are selected for the DMS-benzene. The $\mathrm{S} \cdots \pi$ contact distances and the corresponding interaction energies are obtained for those geometries. The basis set superposition error (BSSE) is corrected to the interaction energy using the counterpoise method of Boys and Bentardi employing the Massage keywords. ${ }^{42}$ The $S \cdots \pi$ interaction energy is denoted with $\Delta E_{\mathrm{BSSE}}$ after BSSE correction.

\section{Results and Discussion}

DMS Monomer: Before the analysis about the DMSbenzene complex. it is necessary to know molecular properties of the DMS monomer. To examine the applicability of the selected theoretical methods, three different conformers of the DMS have been optimized using MP2 and B3LYP methods with $6-311+\mathrm{G}^{*}$ basis set. Three conformers are denoted by $\mathrm{C}_{y}$. $\mathrm{C}_{\mathrm{s}}$. and $\mathrm{C}_{y}$ ' $^{\prime}$ according to their symmetries as seen in Figure 1, along with the atomic numbering for the $\mathrm{C}_{\hat{y}}$ confonmer of the DMS which is also applicable to the other two conformers of the DMS and the corresponding conformers of the FDMS. Figure 1 shows the molecular electrostatic potential (MESP) of the DMS and FDMS. The primary structural parameters, the natural bond orbital (NBO) populations of the $\mathrm{S}$ atom. and the relative energies of the three conformers of the DMS are represented in Table 1 .

From the structural parameters and the relative energies for the three conformers of the DMS. the following three points can be drawn. First of all, the structural parameters obtained using MP2 and B3LYP methods with $6-311+\mathrm{G}^{*}$ 
Table 1. MP2 and B3LYP predicted geometrical parameters, NBO Charge of the sulfur atom $(q(S))$, and relative Energies $\left(E_{\text {rel }}\right)$ for three confonners of the DMS

\begin{tabular}{|c|c|c|c|c|c|c|c|c|}
\hline \multirow[b]{2}{*}{ Method } & \multicolumn{4}{|c|}{$\operatorname{DMS}\left(\mathrm{C}_{Y}\right)$} & \multicolumn{2}{|c|}{$\operatorname{DMS}\left(\mathrm{C}_{i}\right)$} & \multicolumn{2}{|c|}{$\operatorname{DMS}\left(\mathrm{C}_{14}\right)$} \\
\hline & MP2 & B33LYP & Theo." & Exp." & MP2 & B3LYP & MP2 & BSLYP \\
\hline$T(S 1-C 2)$ & 1.803 & 1.823 & 1.803 & 1.807 & 1.805 & 1.826 & 1.816 & 1.838 \\
\hline $\mathrm{r}(\mathrm{C} 3-\mathrm{H} 9)$ & 1.092 & 1.092 & 1.092 & 1.116 & 1.090 & 1.090 & 1.090 & 1.090 \\
\hline $\mathrm{r}(\mathrm{H} 6-\mathrm{H} 9)$ & 2.682 & 2.760 & & & 2.572 & 2.642 & 2.132 & 2.186 \\
\hline$\angle(\mathrm{CSC})$ & 98.19 & 99.49 & 97.09 & 9905 & 99.64 & 100.75 & 102.25 & 102.80 \\
\hline$\angle(\mathrm{SlC} 3 \mathrm{H} 5)$ & 107.72 & 107.08 & 110.04 & 109.30 & 109.69 & 109.82 & 110.27 & 110.25 \\
\hline$E_{[\mathrm{e}]}$ & 00 & 0.0 & & & 2.09 & 1.89 & 4.36 & 3.78 \\
\hline$q(S)$ & 0.192 & 0.171 & & & 0.177 & 0.156 & 0.167 & 0.146 \\
\hline
\end{tabular}

"Reference 43 . "Reference 44 . Bond lengths are in $\AA$. bond angles in degrees, energies in kcalmol, and clarges in $\mathrm{e}^{+}$. Calculations were done with 6 $311+\mathrm{G}^{*}$ basis set.

basis set are in reasonably good agreement with the previous theoretical ${ }^{4 \hat{j}}$ and experimental ${ }^{44}$ results. This demonstrates that these two methods are all reliable for the geometry optimization. Taken the energy of the most stable conformer $\left(\mathrm{C}_{2 v}\right)$ as the reference (zero). the relative energies of the three conformers are obtained. Secondly. the relative energies obtained using MP2 and B3LYP methods are somewhat different. This demonstrates that the interaction energy calculated using B3LYP method is unreliable. which is due to the inherent lack of describing the dispersion energy in B3LYP calculations as addressed in a previous report. ${ }^{30}$ Nevertheless. it should be mentioned that the trend of the relative energies for the different conformers are the same in both MP2 and B3LYP results. Hereafter. our discussion will be given based on the MP2/6-311+G* calculations, otherwise specified. The $\mathrm{C}_{\mathrm{ar}}$ conformer is the most stable among the three conformers, which is predominantly arisen from the smallest $\mathrm{H} \cdots \mathrm{H}$ steric repulsion caused by the longest $\mathrm{H} 6$ $\cdots \mathrm{H} 9$ contact distance of $2.682 \AA$. The $\mathrm{C}_{2 \mathrm{r}}$ ' conformer has the highest energy due to the largest $\mathrm{H} \cdots \mathrm{H}$ steric repulsion caused by the shortest $\mathrm{H} 6 \cdots \mathrm{H} 9$ contact distance of $2.132 \AA$. The increase of the $\mathrm{H}^{\cdots} \mathrm{H}$ steric repulsion can be recognized by the enlarged bond angle of $\angle(\mathrm{CSC})$ and $\angle(\mathrm{SIC} 3 \mathrm{H} 5)$, as can be noted in Table 1. It should be noted that the NBO charges of the $S$ atom for the $C_{s}$ confonmer is less positive than that of the $\mathrm{C}_{\hat{y}}$ conformer. while more positive than that of the $\mathrm{C}_{2} y^{\prime}$ conformer. This should be useful in speculating the $S \cdots \pi$ interaction strength. We expect that the existence of the $S \cdots \pi$ interaction should be possible because the NBO charges of the $\mathrm{S}$ atom in DMS are positive in all the three conformers. while the NBO charge of the $\mathrm{S}$ atom in $\mathrm{H}_{2} \mathrm{~S}$ is negative, -0.251 at MP2/6-311+ $\mathrm{G}^{*}$ calculations. Apparently. based on the NBO charges. it seems to be reasonable to choose DMS rather than $\mathrm{H}_{2} \mathrm{~S}$ as a model to investigate the $\mathrm{S}$ $\cdots \pi$ interaction in proteins. However. when we calculated the molecular electrostatic potential. we realized that even the DMS would not give the $S \cdots \pi$ interaction with benzene. As seen in Figure 1, the MESP is completely negative around the sulfur atom. thus DMS looks infeasible to have the $S \cdots \pi$ interaction with benzene in which $\pi$-electron clouds render strong negative MESP above and below the benzene plane. This expectation can be observed during the
Table 2. MP2 and B3LYP predicted geometrical parameters, NBO Charge of the sulfur atom ( $(\mathrm{g} S)$ ), and relative energies $\left(E_{\text {rel }}\right)$ of the FDMS

\begin{tabular}{|c|c|c|c|c|c|c|}
\hline \multirow[b]{2}{*}{ Method } & \multicolumn{2}{|c|}{ FDMS $\left(C_{2 v}\right)$} & \multicolumn{2}{|c|}{$\operatorname{FDMS}\left(C_{i}\right)$} & \multicolumn{2}{|c|}{$\operatorname{FDMS}\left(\mathrm{C}_{2 v^{\prime}}\right)$} \\
\hline & MP2 & B3LYP & $\mathrm{MP} 2$ & B3LYP & MP2 & BiLYP \\
\hline$r(S 1-C 2)$ & 1.816 & 1.839 & 1.816 & 1.839 & 1.833 & 1.858 \\
\hline r(C3-F9) & 1.333 & 1.338 & 1.336 & 1.341 & 1.337 & 1.342 \\
\hline$r(F 6-F 9)$ & 2.804 & 2.882 & 2.718 & 2.797 & 2.460 & 2.488 \\
\hline$\angle(\mathrm{CSC})$ & 99.23 & 100.33 & 100.24 & 101.38 & 107.11 & 107.11 \\
\hline$\angle(\mathrm{SlC} 3 \mathrm{~F} 5)$ & 106.04 & 105.71 & 113,07 & 113.23 & 115.78 & 115.57 \\
\hline$E_{1 \leqslant 1}$ & 00 & 0.0 & 1.48 & 1.50 & 7.11 & 5.89 \\
\hline$q(S)$ & 0.183 & 0.170 & 0.171 & 0.161 & 0.150 & 0.140 \\
\hline
\end{tabular}

Bond lengths are in $A$, bond angles in degrees, energies in kcal mol, and charges in $\mathrm{e}^{-}$. Calculations were done with $6-311-\mathrm{G}^{*}$ basis set.

geometry optimization of the DMS-benzene dimmer, which will be discussed in the next section. To make the $\mathrm{S} \cdots \pi$ interaction occur in the complexation with benzene, we chose the fully fluorinated DMS (FDMS) expecting that the electronegative fluorines cause the positive MESP around the sulfur atom. We obtained the expected results as seen in Figure 1. Therefore in the complexation with DMS. electronegative aromatic ring center may prefer to interact with the electropositive methyl groups. while in the complexation with FDMS, it may prefer to interact with the sulfur atom.

The structural parameters. NBO charges of the $\mathrm{S}$ atom. and relative energies of the FDMS conformers are represented in Table 2. It is noted that there is no significant differences in the stnuctural parameters in MP2 and B3LYP results. while the relative energies are quite different in MP2 and B3LYP results. as noted in the DMS calculations. Similarly to DMS, the stability of the conformers of the FDMS is in the following order: $\mathrm{C}_{y v}>\mathrm{C}_{s}>\mathrm{C}_{y}$ '. The significant difference in the $\mathrm{F} 6 \cdots \mathrm{F} 9$ contact distances is responsible for the significant difference in repulsions between the two $\mathrm{F}$ atoms. which determines the relative stability of the conformers of the FDMS. Similarly to the DMS. the NBO charge of the $\mathrm{S}$ atom of the $\mathrm{C}_{2 \mathrm{r}}$ conformer is larger than those of the other two confonmers. The MESP shape of the FDMS is completely different from that of the DMS as seen in Figure 1. In the FDMS, the electronegative 


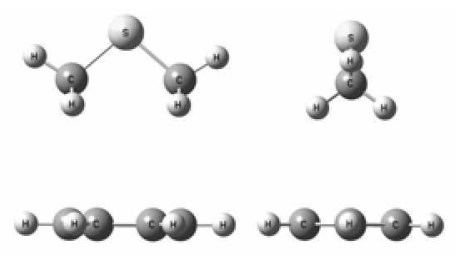

$C_{2 v} \mathbf{I}$

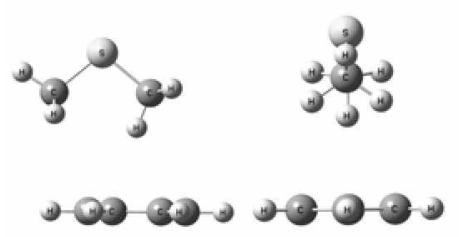

$C_{s} \mathbf{I}$
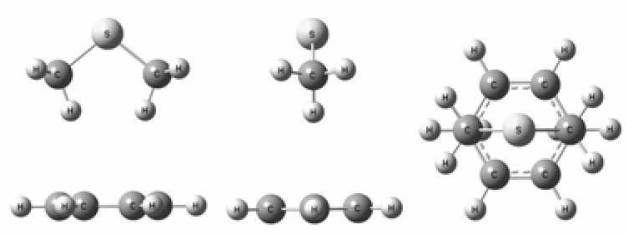

$C_{2 v}{ }^{\prime} \mathbf{I}$
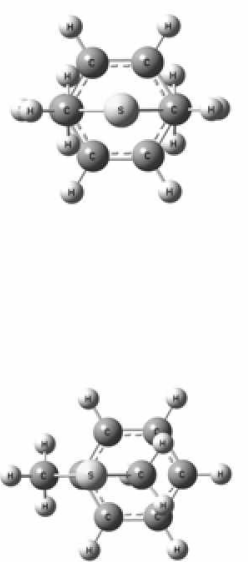

$-\infty-30-0$

$C_{s}$ II

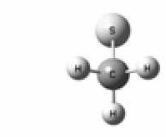

$.00-30.0000$

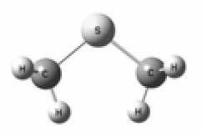

$C_{2 v}$ ' II

Figure 2. MP2/6-311+G* optimized structures of the six binding modes of the DMS-benzene complex.

potential is forned around the trifluoromethyl groups due to the electronegative fluorine atoms, while the electropositive potential compasses the $S$ atom. Thus, the FDMS-benzene complex can have the $\mathrm{S} \cdots \pi$ interaction. while the DMSbenzene may not.

DMS-Benzene and FDMS-Benzene Complexes. There can be several configurations (interaction modes) for the DMS-benzene complexes. According to the symmetry, we denote three different binding modes. $\mathrm{C}_{\hat{4}} . \mathrm{C}_{\mathrm{s}}$, and $\mathrm{C}_{2 \mathrm{Y}}$ as seen in Figure 2. In $\mathrm{C}_{2 k}$, four hydrogen atoms (two hydrogen atoms from each methỵl groups) are interacting with benzene $\pi$ electrons. In $C_{\text {s. }}$ three hydrogen atoms (one from one methyl and two from the other methyl group) are interacting with benzene. For $\mathrm{C}_{34}$ ', two hydrogen atons (one from each methyl groups) are interacting with benzene. Each interaction mode can be classified into two different modes. I and II. For the mode I. The C-S-C plane of the DMS bisects the benzene through the two carbon atoms. while it bisects the benzene through the centers of two $\mathrm{C}-\mathrm{C}$ bonds for the mode II as seen in Figure 2. The MP2 optimized structures for the six different binding modes for the DMSbenzene were shown in Figure 2. Based on these binding modes. we denote six structures as X I and X II ( $\mathrm{X}=\mathrm{C}_{3 \mathrm{v}}$. $\mathrm{C}_{\mathrm{s}}$. and $\mathrm{C}_{34^{\prime}}$ ). They are distinguished each other by the orientation of the DMS and benzene molecule. As we described. the six interaction modes are selected from the fact that the benzene should interact with methyl groups of the DMS based on its MESP.

The intermolecular distances between the DMS and the
Table 3. MP2/6-311+G*alculated intermolecular distances $(\mathrm{I}(\mathrm{S} \cdots \pi)$ ) and interaction energies $(\Delta \mathrm{F})$ for the DMS-Benzene

\begin{tabular}{|c|c|c|c|c|c|c|}
\hline & $C_{I Y} \mathbf{I}$ & $\mathrm{C}_{Z_{Y}} \boldsymbol{\Pi}$ & $C_{s i s} \mathbf{I}$ & $C_{s}$ II & $C_{y_{Y}^{\prime}}^{\prime} \mathbf{I}$ & $\mathrm{C}_{2}{ }^{\prime} \mathbf{I I}$ \\
\hline$\Gamma(S \cdots \pi)$ & 4.831 & 4.790 & 4.696 & 4.712 & 4.895 & 4.827 \\
\hline$\Delta \mathrm{E}$ & -6.44 & -6.54 & -5.32 & -4.77 & -2.82 & -2.93 \\
\hline$\Delta \mathrm{E}_{\mathrm{BSSE}}$ & -3.73 & -3.74 & -1.86 & -1.43 & 0.31 & 0.31 \\
\hline
\end{tabular}

Bond lengths are in $\AA$. energies in kcalimol. $\Delta E$ and $\Delta E_{\text {BSSE }}$ denote the interaction enercies without and with basis sets superposition enror (BSSE) correction, respectively.

benzene is defined by the distance between the sulfur atom and the center of the benzene ring. and is denoted by $\mathrm{r}(\mathrm{S} \cdots \pi)$. The intermolecular distances and the interaction energies for the six different interaction modes are listed in Table 3. It should be noted that the interaction energies of the DMS-benzene for the interaction modes I and II are very similar. The interaction energies are also very similar for the $\mathrm{C}_{3 \mathrm{y}}$ and $\mathrm{C}_{3 y}{ }^{\prime}$ conformers. and they are slightly different from that of the $C_{s}$. The intermolecular distance $r(S \cdots \pi)$ ranges from 4.696 to $4.895 \AA$ for the six interacting modes. The main origin of the interaction energy variation for the six binding modes is attributed to the intrinsic nature of the internal rotational barrier in DMS. In other words. for the DMS molecule, the relative energies of the $C_{s}$ and $C_{2 y}$ ' confomers is 2.09 and $4.36 \mathrm{kcal} / \mathrm{mol}$. respectively. and which is the intrinsic intenal rotational barrier in DMS. Considering only the rotational barrier of DMS and assuming the intermolecular interaction for the DMSbenzene complex is constant regardless of the orientation. 
the interaction energies for the $\mathrm{C}_{\mathrm{s}} \mathrm{I}$ and $\mathrm{C}_{2 \mathrm{y}}$ ' I should be 2.09 and $4.36 \mathrm{kcal} / \mathrm{mol}$ higher than that for the $\mathrm{C}_{2 \mathrm{v}} \mathrm{I}$. respectively. On the other hand, in our calculation. it was found that the interaction energies for the $\mathrm{C}_{S} \mathbf{I}$ and $\mathrm{C}_{2} v^{\prime} \mathrm{I}$ are higher than that for the $\mathrm{C}_{x} \mathrm{I}$ by 1.87 and $4.04 \mathrm{kcal} / \mathrm{mol}$. respectively. Therefore. it suggests that the interaction energy for the DMS-benzene may depend on the orientation though it is mainly determined by the stability of the DMS monomer.

It has been reported that the $S \cdots \pi$ distance in the optimized configuration of methanethiol-benzene system is $4.4 \AA$. The interaction energy for this geometry was reported to be $-3.0 \mathrm{kcal} / \mathrm{mol}$ at $\mathrm{MP} 2 / 6-31 \mathrm{G}^{*} / / \mathrm{HF} / 3.2 \mathrm{lG} *$ level. ${ }^{17} \mathrm{In}$ a recent paper, ${ }^{\hat{2} 1}$ the investigation illustrates that the $S \cdots \pi$ distance in the lowest energy conformation was only $3.73 \AA$. and the corresponding interaction energy was $-3.71 \mathrm{kcal} /$ mol. The conformation they reported does not necessarily correspond to the most possible structure in real biological systemis. Table 3 demonstrates that the $\mathrm{S} \cdots \pi$ contact distance ranges from 4.70 to $4.90 \AA$ for the sulfur containing amino acids and the aromatic rings. This distance is slightly longer than those reported in previous papers. which might be caused by steric repulsion. The interaction energy $(\Delta \mathrm{E})$ changes distinctly with the change of the DMS structure. ranging from -6.54 to $-2.82 \mathrm{kcal} / \mathrm{mol}$ without basis sets superposition error (BSSE) correction. This value decreases significantly with the BSSE correction, indicating the importance of the BSSE correction for the $S \cdots \pi$ interaction energy. though the qualitative analysis gives the same results. The most stable complex ( $\mathrm{C}_{2}$ II) has the BSSE corrected interaction energy $\left(\Delta \mathrm{E}_{\mathrm{BSSS}}\right)$ of $-3.74 \mathrm{kcal} / \mathrm{mol}$. which is in good agreement with the previous investigation. ${ }^{21}$ It is well known that a gas-phase interaction energy between oppositely charged structures may be reduced as greatly as $-100 \mathrm{kcal} / \mathrm{mol}$ in aqueous solution. ${ }^{45}$ Although the $\mathrm{S} \cdots \pi$ interaction tends to occur in the interior of proteins and is thus not subject to such a dramatic solution effect, this interaction should be reduced dramatically for the opposite $S^{s+}-\pi^{s-}$ charges. Even though the interaction energies were reduced to $-3.74 \mathrm{kcal} / \mathrm{mol}$. these $\Delta \mathrm{E}$ should be significant for the determination of protein folding.

The substituent effect on the $S \cdots \pi$ interaction is clearly reflected in the structures shown in Figure 3. It can be clearly seen that the DMS rotates $\left(\sim 30^{\circ}\right)$ and slides off to have the methyl group interact with the $\pi$-electron clouds of the benzene due to the high $\sigma$ charge density of the methyl
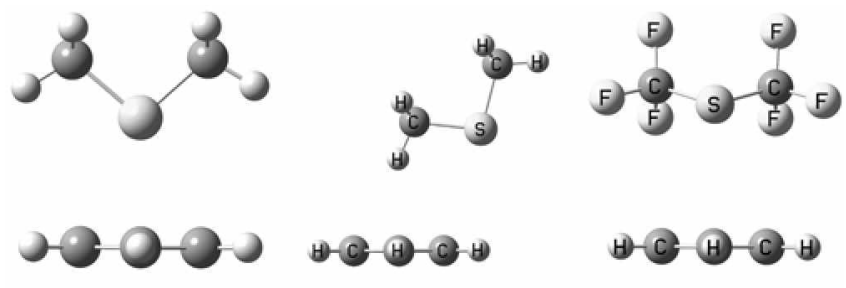

Initial Conformer

DMS-Benzene

FDMS-Benzene

Figure 3. The DMS-benzene and FDMS-benzene complexes pursuing the $S \cdots \pi$ interactions.
Table 4. MP2/6-31 I+G* calculated intermolecular distance $(\mathrm{r}(\mathrm{S} \cdots \pi)$ ) and interaction energy $(\Delta \mathrm{E})$ for the DMS-benzene and the FDMSbenzene

\begin{tabular}{lcc}
\hline & DMS-benzene & FDMS-benzene \\
\hline $\mathrm{r}(\mathrm{S} \cdots \pi)$ & 3.942 & 3.274 \\
$\Delta \mathrm{E}$ & -7.94 & -7.68 \\
$\Delta \mathrm{E}_{\mathrm{BSSE}}$ & -4.34 & -2.09 \\
\hline
\end{tabular}

Bond lengths are in $\AA$. energies in kcal mol. $\Delta E$ and $\Delta E_{B S S E}$ denote the interaction energies without and with basis sets superposition error (BSSE) correction, respectively:

group. This should be reasonable with the reference of MESP maps described in Figure I because the methyl group of DMS having positive MESP prefers to combine with the $\pi$ electron of benzene ring. Thus, it looks more likely to be the $\mathrm{H} \cdots \pi$ interaction rather than the $\mathrm{S} \cdots \pi$ interaction between the DMS and the benzene. Thus, we try to investigate how the structure changes when it is initially given as a conformation where the $\mathrm{S}$ atom of the DMS is directly interacting with the benzene $\pi$ electrons pursuing the $S \cdots \pi$ interaction. Figure 3 shows the initial structure of both the DMS-benzene and the FDMS-benzene, and their optimized structures obtained by the MP2/6-311+G* calculations. For the optimized DMS-benzene, the $\mathrm{H} \cdots \pi$ interaction is still dominating. while the sulfur atom also aids the interaction by $\mathrm{S} \cdots \mathrm{H}-\mathrm{C}$ interaction. However, for the optimized FDMSbenzene complex, the $S$ atom is located over the benzene ring. and it should be regarded as the $\mathrm{S} \cdots \pi$ interaction between the FDMS and benzene. This is exactly what we expected from the MESP of the FDMS where the vicinity of the $S$ atom has positive MESP. Therefore. the effect of the substituent group on the $S \cdots \pi$ interaction is remarkable.

The intermolecular distances and the interaction energies for the DMS-benzene and the FDMS-benzene complex are listed in Table 4. The intermolecular distance in the DMSbenzene is larger than that in the FDMS-benzene by $0.67 \AA$ because the negative MESP around the sulfur atom of the DMF causes the significant repulsion between the sulfur and $\pi$-electron clouds of the benzene. But, it should be noted that this binding mode is favored than any of the six binding modes in Figure 2 due to the additional $\mathrm{S} \cdots \mathrm{H}-\mathrm{C}$ interaction. While the interaction energy of the FDMS-benzene complex is comparable to that of the DMS-benzene without the BSSE correction, the BSSE corrected interaction energy of the FDMS-benzene is substantially decreased. This may be the result of partial $\mathrm{F} \cdots \pi$ repulsion and the steric hindrance. It is very interesting to note that the $S \cdots \pi$ interaction mode does exist in the FDMS-benzene. It implies that the $S \cdots \pi$ interaction can be found in many biological systems depending on the substituents. The reliability of B3LYP method in the calculation of the $S \cdots \pi$ interaction energy has been investigated. Taken the optimized geometry with B3LYP method as an initial structure. the optimization was performed with MP2 method. It was found that the MP2 predicted interaction energy increased significantly $(-7.94 \mathrm{kcal} / \mathrm{mol}) \mathrm{com}-$ pared with that obtained with B3LYP method $(-0.70 \mathrm{kcal} /$ mol). while the structural change was small. Thus. it can be 
safely drawn that B3LYP calculation is unreliable in the calculation of the $S \cdots \pi$ interaction, as the B3LYP calculation is often criticized in weak interaction calculations such as the $\mathrm{H} \cdots \pi$ and the $\pi \cdots \pi$ stacking interactions. Nevertheless. the B3LYP calculation would give reliable geometries for sulfur bearing and aromatic containing amino acids, thus it can be useful in rough and quick estimation of the chemical and electronic properties that mainly depend on the structures.

\section{Conclusion}

The $\mathrm{H} \cdots \mathrm{H}$ steric repulsion contributes predominantly to the stability of DMS and the NBO charge population on the $\mathrm{S}$ atom. The effect of nucleophilic group on DMS is conspicuous for the distinct variation of NBO charge and MESP population. and the interaction mode between aromatic ring and DMS also changes on the electronegative substitution from $\mathrm{H}^{\cdots} \pi$ to $S \cdots \pi$ coupling. Through the investigation on the $S-\pi$ contact distance and the corresponding interaction energy for DMS-benzene complex, it is found that the DMS structure plays a key role in determination of the sulfur atom bearing amino acids and aromatic side chains of certain amino acids $(\mathrm{r}(\mathrm{S} \cdots \pi))$ and the corresponding interaction energy $(\Delta \mathrm{E})$. Both MP2 and B3LYP methods can be used to optimize the geometry structure of sulfur contained anino acid and aromatic ring compounds, but the $S \cdots \pi$ interaction energy calculation obtained with B3LYP method is not reliable.

Acknowledgment. This paper was supported by the Bioinformatics Center at Korea Institute of Science and Technology Information. 2006. We also acknowledge the Supercomputing Center at Korea Institute of Science and Teclunology' Information.

\section{References}

1. Dill K. A. Biochemishy 1990, 29,7133

2. (a) Myung, P.-K.: Park K.-Y.: Sung, N.-D. Buhl Kor them. Soc. 2005, 26, 1941. (b) Donnene. C.: Bond. P. J: Deol. S. S.; Sansom. M. S. P. J. Am. Chent Soc. 2003. 125. 14966. (c) Bujalowski. W. Chem. Rev. 2006. 106. 556. (d) Lee. J.-Y: Kim. Y. Bull. Kor. Chem. Soc. 2005. 26.1695 (e) Jeong. M. S.: Jang. S. B. Bull. Kor. Chem. Soc. 2006. 27. 87

3. Viguera. A. R.: Serrano. L. Biochemishy $1995,34,8771$

4. (a) Morgan. R. S.: Tatsch, C. E.; Gushard. R. H.: McAdon. I. M.: Warme. P. K. Int. J. Pentide Protein Res. 1978. 11. 209. (b) Morgan. R. S.: McAdon. J. M. Int. J. Pettide Protein Res. 1980. 15. 177.

5. Rodner. B. L.: Jackman. L. M.: Morgan. R. S. Biochem. Biophns. Res. Commom. 1980. 94. 807.

6. Shortle D.: Meeker. A. K. Protems Struct. Funct. Genet. 1986. 1, 81.

7. Lebel, M: Sugg. E. E.: Hruby. V. J. hit. J. Peptide Protein Res. 1987. 29.40 .

8. Shortle. D.: Meeker. A. K.: Freire. E. Biochentistry 1988. 27, 4761

9. Shortle. D.: Stites. W. E.: Meeker. A. K. Biochentistry 1990. 29.8033.

10. Yamaoutsu, N.: Morguchi, I.: Hirono. S. Biochim Biophns. Acta $1993,1203,243$.

11. Yamaoutsu, N.: Moriguehi, I.: Kollnan. P. A.: Hirono. S. Biochim. Biophss. Acta 1993. 1163.81.

12. Kimura. T.: Furukawa. N. Tetrahedron Lett. 1995. 36. 1079.
13. Viguera. A. R.: Serrano. L. Biochentistry 1995. 34. 8771.

14. Spencer. D. S.: Stites. W. E. J. Mol Biol 1996. 257. 497.

15. Gauss. T.: Stanton. I. F. J. Phis Chent A 2000 . 104. 2865.

16. Nemethy, G.; Scheraga. H. A. Biochem. Biophus. Res. Commun. $1981.98,482$

17. Cheney, B. V.: Schulz, M. W.: Cheney, J. Biochim Biophys. Actat. 1989.996.116.

18. Munnoz. V.: Serrano. L. Protems Struct. Fimct. Genet. 1994. 20.301

19. Munoz. V.: Serran1o. L. J. Mol. Biol. 1995. 2+5. 275

20. Pranata, J. Bioorg. Chem. 1997. 25, 213

21. Duan. G. L.; Smith, V. H., J.: Weaver, D. F. Mol. Phys. 2001. 99 1689.

22. Tauer. T. P.: Derrick. M. E.: Sherrill. C. D. J. Phws. Chent. A 2005. 109. 191.

23. Reid. K. S. C.: Lindley. P. F.: Thornton. I. M. FEBS Lett. 1985. 190. 209 .

24. Allen. F. H.: Kennard, O. Chem. Design Auto Kew's 1993. 8. 1 \& 31.

25. Zauhar. R. J: Colbert. C. L: Morgan. R. S.; Welshm, W. J. Biopolymers 2000. 53. 233.

26. Allet1. F. H. Acta Cyrstallog: B 2002. 58.380

27. Calvert. J. G.: Pitts. J. N.. Ir. Photochentisty. Wiley: New York. 1966.

28. Thompson, S. D: Carrol, D. G; Watson. F.: O'Donnell. M. MoGlynn, S. P. J. Chem. Phus. 1966, 45. 1367.

29. Charlson, R. J.: Lovelock. J. E; Andreae. M. O; Warren. S. G. Nature 1987.326.655.

30. Bates. T. S.: Lamb. B. K.: Guenther. A.: Dignon. T.: Stoiber. R. E. J. Atmos. Chem. 1992. It.315.

31. Manaa. M. R.: Yarkony, D. R. J. Am. Chem. Soc. 1994. 116, 1144.

32. Haya. B. M: Quintana. P.: Bañares. L.; Samartzis, P.: Smith. D. J.; Kitsopoulos, T. N. J. Chem. Phys. 2001, 114.4450.

33. Mousavipour. S. H.: Emad. L.: Fakhraee S. J. Phns. Chem. A 2002. 106. 2489.

34. Bernstein. F. C.: Koetzle. T. F.: Willians. G. J. B.: Meyer. E. F.: Brice. M. D; Rodgers, J. R.: Kennard. O.: Shimanouchi, T: Tasumi, M. J. 1 Kol. Biol. 1977. 112,535.

35. Solimannejad, M.: Pejov, L. J. Phus. Chem. \& 2005, 109, 825.

36. (a) Kim. K. S.: Tarakeshwar. P.: Lee. T. Y. Chent. Rev: 2000. 100. 4145. (b) Vaupel. S.: Brutschy. S.: Tarakeshwar. P.: Kiml. K. S. J. Ant Chem. Soc. 2006. 128. 5416. (c) Lee. E. C.: Hong. B. H.: Lee. J. Y.: Kim, J. C.: Kim, D.; Kim. Y: Tarakeshwar, P.; Kim. K. S. $d$ Am. Chem. Soc 2005, 127,4530. (d) Tarakeshwar. P.: Choi. H. S.; Kim. K. S. J. Am. Chem Soc. 2001, 123.3323.

37. (a) Hong. B. H.: Lee. T. Y.: Cho. S. T.: Yun1. S.: Kim. K. S. J Org. Chem. 1999. 6t. 5661 (b) Tarakeshwar. P.: Lee. S. J.: Lee. J. Y.: Kim. K. S. J. Chem. Phss 1998. 108.7217. (c) Ren1. T.: Jin. Y. Kim. K. S.: Kim. D. H. J. Bionol Struct. Dynamics 1997. 15, 401.

38. (a) Gotch, A. J.: Zwier, T. S. J. Chem. Phys. 1992, 95,3388 . (b) Augspurger, J. D.: Dikstra. C. E.: Zwier. T. S. J. Plys. Chem. 1992. 96. 7252. (c) Gutowsky. H. S.: Emilsson. T.: Arunan. E. $J$ Chent. Phus 1993. 99. 4883. (d) Arutant. E.: Emilsson. T.: Gutowsky. H. S. J. Chem. Phs. 1994. 101. 861. (e) Arunant. E.: Emilsson, T.: Gutowsky. H. S.: Fraser. G. T.: Oliveira. G. de: Dykstra, C. E. J. Chem. Plys. 2002. 117,9766.

39. (a) Lee, J. Y: Kim, J:- Lee. H. M: Tarakeshwar. P. Kim. K. S. $d$. Chent Phys 2000. 113.6160. (b) Tarakeshwar. P.: Kiml. K. S.: Brutschy. B. J. Chen. Phws. 2000. 112. 1769.

40. (a) Tarakeshwar. P: Choi. H. S.: Lee. S. T.: Lee J. Y: Kim. K. S.: Ha. T.-K.; Jang, J. H.; Lee, J. G.: Lee. H. J. Chem. Phys. 1999, 11 . 5838. (b) Kim. K. S.; Lee. J. Y.; Choi, H. S.; Kim. J.: Jang, J. H. Chem. Phis. Lett. 1997. 265, 497.

41. Frisch. M. J. et al. Gaussiam 03. Revision Al: Gaussian Inc: Pittshurgh. PA. 2003

42. Boys. S. F.: Bernardi. F. Mol Plns 1970. 19. 553

43. Mousavipour. S. H; Emad. L; Fakhraee, S. J. Plws. Chem. At 2002. 106,2489 .

44. Handbook of Chemistry and Plysics. $80^{\text {th }}$ Ed; Lide, D. R., Ed: CRC Press: Boca Raton. 1999-2000

45. Saigal. S.: Pranata. T. Bioorg. Chent 1997. 25. 11. 